\title{
Eine einfache, spezifische und schnelle Silicium-Bestimmungsmethode im Harn mit dem Drei-Kammer-Bogenbrenner
}

\author{
Von K. RÖTGER ${ }^{1}$ ) \\ Aus der Abteilung.für Medizinische Physik an der Universitäts-Hautklinik Gießen (Leiter: Prof. Dr. R. Herrmann)
}

(Eingegangen am 20. Mai 1966)

Es wird eine Siliciumbestimmungsmethode im unverdünnten und nicht enteiweißten Harn mit Hilfe des Drei-Kammer-Bogenbrenners angegeben. Als Anwendungsbeispiel werden Vergleichsmessungen an zwei verschiedenen Kollektiven von Probanden durchgeführt.

A method is reported for the determination of silicon in undiluted urine, without removal of protein, with the aid of the three-chamberplasma-arc. Its application is illustrated by comparative measurements on two different groups of probands.

In einer früheren Arbeit (1) wurde der Drei-KammerBogenbrenner für klinisch-chemische Untersuchungen vorgeschlagen. Mit seiner Hilfe können „flammenphotometrische Untersuchungen" mit hohen Temperaturen durchgeführt werden. Diese Antegungsquelle ist besonders dann empfehlenswert, wenn schwerer anregbare oder dissoziierbare Elemente analysiert werden sollen. In einer zweiten Arbeit (2) wurde als erstes Anwendungsbeispiel eine Lipoidphosphorbestimmungsmethode im Blutserum mit Hilfe dieser Anregungsquelle ausgearbeitet. In der hier folgenden Arbeit soll nun eine einfache, spezifische und schnelle Methode zur Bestimmung des Siliciums im unverdünnten und nicht enteiweißten Harn mit Hilfe der gleichen Bogenanordnung angegeben werden.

Silicium kommt in fast allen Organen des menschlichen Körpers, meist als Kieselsäure $\mathrm{SiO}_{2}$, vor. E. RuF beschreibt z. B. eine photometrische Methode zur Bestimmung von Phosphor und Kieselsäure in Lösungen, die auf der Bildung von Molybdatkomplexen (Farbreaktion) beruht (3). Eine einfache spektroskopische Siliciumbestimmungsmethode im Harn unter Verwendung von spezifischen Atomlinien und direktem Einstäuben der Probenflüssigkeit in die Anregungsquelle wurde uns nicht bekannt. Mit den in klinischchemischen Laboratorien verbreiteten normalen flammenphotometrischen Analysenmethoden bei den relativ niedrigen Flammentemperaturen läßt sich Silicium in den vorkommenden Konzentrationen im Harn direkt durch Atomemission nicht nachweisen.

\section{Methodik}

\section{Probenvorbereitung}

Eine chemische Vorbereitung der Harnprobe ist nicht erforderlich. Der Harn wird, um Zersetzungs- und Entmischungserscheinungen zuvorżukommen, möglichst bald nach Abgabe durch den Patienten durch direktes Einstäuben in das heiße Plasma gemessen.

Pro Messung werden $0,2 \mathrm{~m} l$ Harn benötigt.

\section{Apparat und Betriebsbedingungen}

Als Anregungsquelle dient der in (1) bereits beschriebene DreiKammer-Bogenbrenner. Die im Plasma angeregten Atome und Ionen emittieren Linien, deren Wellenlänge charakteristisch für das emittierende Element, und deren Intensität in der Regel ein Maß für die Konzentration dieses Elementes im Plasma, und nach entsprechender Eichung auch in der Analysenlösung, ist. Die vom Bogenbrenner ausgehende Strahlung wird in einem, ,Beckman-DUG2400-Spektralphotometer" mit einem Quarzprisma als Mono-

1) Unterstützt mit Mitteln der Deutschen Forschungsgemeinschaft. chromator und einem 1 P 28 PEV von der Fa. RCA als Strahlungsempfänger zerlegt und verstärkt. Der eingebaute Gleichstromverstärker sorgt für eine Nachverstärkung und ein angeschlossener Honeywell-Schreiber registriert das Ergebnis. Die unten angegebenen Eich-, Analysen- und Blindlösungen werden mittels eines "Beckman-Zerstäubers" durch eine Bohrung in der Anode als feiner Nebel direkt in das Plasma eingebracht.

Die Betriebsdaten des Bogenbrenners bei unseren Silicium-Analysen waren: 24 A Stromstärke bei einer Bctriebsspannung von etwa $60 \mathrm{~V}$ und einem Gesamtargondurchsatz von 47,5 l/Min. Weil der Harn in unverdünntem und nicht enteiweißten Zustand zerstäubt werden sollte, mußten wir, um der Bildung von Koagula an der Zerstäuberspitze entgegenzuarbeiten, einen relativ hohen Zerstäubergasdruck von $1,1 \mathrm{~kg} / \mathrm{cm}^{2}$ wählen, was einem Gasdurchsatz von etwa $3,5 \mathrm{l} / \mathrm{Min}$. entspricht. Die Kühlwasserdurchflußmenge durch unsere Bogenanordnung betrug 1,5 l/Min. Gemessen wurde bei der SiliciumLinie $2516 \AA$, die sich bei den bei uns vorliegenden Versuchsbedingungen als die nachweisempfindlichste Linie herausstellte. Die günstigste Meßhöhe im Beobachtungsfenster des Plasmabogens wurde durch Auftragen des Verhältnisses Linie zu Untergrund in Abhängigkeit von der Meßhöhe entsprechend den PhosphorAnalysen (2) ermittelt und betrug hier $6 \mathrm{~mm}$ über dem unteren Rand der $8 \mathrm{~mm}$ im Durchmesser betragenden Beobachtungsöffnung. Der Eintrittsspalt des Spektralphotometers „Beckman $D U$ " wurde auf $0,02 \mathrm{~mm}$ eingestellt.

\section{Eichung}

Zur Herstellung einer Eichlösung wurden 4,859 g Kieselwolframsäure $\left(\mathrm{H}_{4} \mathrm{Si}\left(\mathrm{W}_{3} \mathrm{O}_{10}\right)_{4}, \mathrm{Fa}\right.$. Merck Nr. 659) in einen 1-l-Kolben gegeben und mit aqua dest. aufgefüllt, was einer Siliciumkonzentration von $50 \mu \mathrm{g} / \mathrm{m} l$ entspricht. Als Blindlösung diente aqua dest. Die zur Aufriahme einer Eichkurve notwendigen variierten SiliciumEichlösungen wurden in gleicher Weise wie oben mit den entsprechenden Mengen an Kieselwolframsäure hergestellt. Die gemessene Silicium-Eichkurve (Abb. 1) war in dem interessierenden Konzentrationsbereich von 1 bis $100 \mu \mathrm{g} / \mathrm{m} l$ linear.

\section{Messung}

Die Bestimmung der Siliciumkonzentration in einer Harnprobe ging so vor sich, daß vor bzw. nach dem Zerstäuben einer Analysenlösung (Harn) je $1 \mathrm{mal}$ die Blindlösung (aqua dest.) und je $1 \mathrm{mal} \mathrm{die} \mathrm{Eichlösung}(50 \mu \mathrm{g} / \mathrm{ml} \mathrm{Si}$ in wäßr. Lösg.) durch die Bohrung in der unteren Elektrode (Anode) in das Plasma eingestäubt und die emittierten Intensitäten bei konstanter Wellenlänge über die Zeit registriert wurden. Die abgelesenen Intensitäten sind wegen des linearen Verlaufs der Eichkurve ein direktes $\mathrm{Maß}$ für die Siliciumkonzentration in der Harnprobe.

Prüfung der Methode

\section{Genauigkeit}

Standardabweichung und prozentualer Fehler wurden nach der in (4) angegebenen Methode ermittelt. Die Standardabweichung $\sigma$ betrug bei 20 Messungen der gleichen Harnprobe bei einem Mittelwert von $51,95 \mu \mathrm{g} / \mathrm{m} l$ $\sigma= \pm 0,75 \mu \mathrm{g} / \mathrm{ml}$, was einem prozentualen Fehler von $\pm 1,5 \%$ entspricht. Die Nachweisgrenze betrug unter 


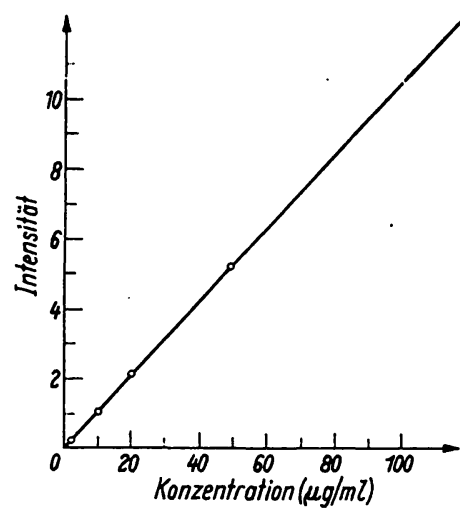

Abb. 1 AufgenommeneSi-Eichkurve (Intensität in rel. Einheiten)

Abb. 2 Zumischversuch (s. Text)

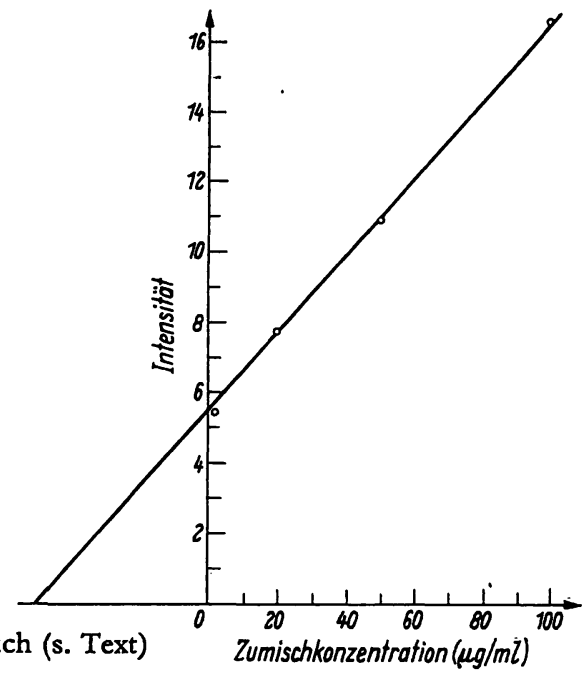

brauch von $0,2 \mathrm{ml}$ entsprach, und $\mathrm{zu}$ jeder Analysenlösung die wäßrigen Blindund Eichlösungen - auch zur Reinigung der Kapillare - zerstäubten.

Anwendungsbeispiel

Zum Schluß geben wir mit der Methode gemessene Siliciumkonzentrationen in Harnproben von zwei Versuchsgruppen an, ohne auf eine medizinische Auswertung dieser Ergebnisse einzugehen.

Wir bestimmten die Siliciumkonzentration im Nachmittagsharn von 26 männlichen Patienten im Alter von 25 bis 45 Jahren der Universitäts-Hautklinik Gießen, bei denen keine Silikoseerkrankungen bekannt waren und die nicht mit

den gewählten Betriebsbedingungen unter Verwendung der Definition von KaISER $(6,4) 0,1 \mu \mathrm{g} / \mathrm{ml}$.

\section{Untergrundstörungen}

Wegen des hohen Auflösungsvermögens des Quarzprismas im UV und der geringen Breite des Eintrittsspaltes (bei $2500 \AA$ und einer Spaltbreite von $0,02 \mathrm{~mm}$ betrug $\Delta \lambda=0,5 \AA$ ) war zu erwarten, daß keine Störungen durch Linien anderer Elemente im Harn in Erscheinung treten würden und $\mathrm{da}$ der Untergrund des Harns identisch mit dem von destilliertem Wasser ist. Dies wurde durch Registrieren über die Wellenlänge bẹ mehreren Harnproben im Vergleich zu Wasser bestätigt.

\section{Richtigkeit}

Außerdem wurde die Richtigkeit der Meßergebnisse dadurch geprüft, daß die in Abbildung 2 gezeigte Zumischkurve aufgenommen wurde. Die durch die $\mathrm{Zu}-$ mischung entstehende Verdünnung (Volumenvergrößerung) wurde bei der Aufnahme des Diagramms berücksichtigt, indem auf das Ausgangsvolumen zurückgerechnet wurde. Der lineare, zur Eichkurve parallele Verlauf der Zumischkurve zeigt, daß keine wesentlichen Ionisations- und ähnliche Störungen in Erscheinung treten. Der Schnittpunkt der Zumischkurve mit der Ordinate gibt, wenn man die Konzentrationsskala beim Schnittpunkt Zumischkurve-Abszisse beginnen läßt, die Si-Konzentration der verwendeten Harnprobe mit $53 \mu \mathrm{g} / \mathrm{ml}$ (gemessen 51,95 $\mu \mathrm{g} / \mathrm{ml}$ ) an. - Eine chemische Vorbereitung der Probe war also nicht nötig. Bei längerem Zerstäuben der Harn-Analysenlösung machte sich allerdings eine langsame Verkrustung des Zerstäubers störend bemerkbar: die Durchflußmenge des zerstäubten Harns wurde im Laufe der Zeit geringer. Diesen Störeinfluß schalteten wir aus, indem wir maximal nur 5 Sek. lang Harn zerstäubten, was einem Probenver- stark siliciumhaltigen Präparaten behandelt wurden. Diesen Meßwerten werden in der folgenden Tabelle die gemessenen Siliciumkonzentrationen von 25 in einer siliciumhaltiges Material verarbeitenden Industrie beschäftigten Arbeitern der gleichen Altersgruppe gegenübergestellt.

\begin{tabular}{|c|c|c|c|c|c|}
\hline \multicolumn{6}{|c|}{ Si-Konzentration der } \\
\hline \multicolumn{3}{|c|}{$\begin{array}{l}\text { Urine von } \mathrm{HK} \text {-Patienten } \\
\mu \mathrm{g} / \mathrm{m} l\end{array}$} & \multicolumn{3}{|c|}{$\begin{array}{l}\text { Urine vọ Industriearbeitern } \\
\qquad \mu \dot{\mathrm{g}} / \mathrm{m} l\end{array}$} \\
\hline $\begin{array}{c}25,0 \\
26,8 \\
31,65 \\
2,16 \\
16,85 \\
57,3 \\
26,05 \\
43,8 \\
4,09\end{array}$ & $\begin{array}{l}23,2 \\
22,7 \\
16,8 \\
18,2 \\
30,0 \\
17,2 \\
28,9 \\
16,3 \\
16,1\end{array}$ & $\begin{array}{c}13,4 \\
27,6 \\
21,5 \\
10,5 \\
8,2 \\
12,5 \\
2,26 \\
35,6\end{array}$ & $\begin{array}{r}130,0 \\
41,4 \\
279,8 \\
50,4 \\
21,8 \\
69,5 \\
10,0 \\
33,3 \\
247,5\end{array}$ & $\begin{array}{r}53,0 \\
74,0 \\
256,0 \\
35,6 \\
153,0 \\
28,1 \\
43,5 \\
34,3\end{array}$ & $\begin{array}{r}239,0 \\
56,5 \\
29,4 \\
17,7 \\
50,0 \\
148,0 \\
39,1 \\
46,1\end{array}$ \\
\hline \multicolumn{6}{|c|}{ Mittelwerte } \\
\hline \multicolumn{3}{|c|}{$\begin{aligned} M_{1} & =21,3 \mu \mathrm{g} / \mathrm{m} l \\
\sigma_{1} & =12,6 \\
n_{1} & =26\end{aligned}$} & \multicolumn{3}{|c|}{$\begin{aligned} M_{2} & =86,7 \mu \mathrm{g} / \mathrm{ml} \\
\sigma_{2} & =83 ; 4 \\
\mathrm{n}_{2} & =25\end{aligned}$} \\
\hline
\end{tabular}

Aus der Tabelle ist ersichtlich, daß der mittlere SiGehalt im Harn bei den Probanden aus dem Si-verarbeitenden Industriezweig deutlich höher liegt als bei der Vergleichsgruppe. Um zu überprüfen, ob der gefundene Mittelwertsunterschied statistisch sicher ist, wird nun ein in (5) genanntes Prüfverfahren auf diese Werte hin angewandt.

Ein echter Unterschied ist sicher, wenn

$$
3 \cdot \sigma_{\text {Diff. }}=3 \cdot \sqrt{\frac{\sigma_{1}^{2}}{\mathrm{n}_{1}}+\frac{\sigma_{2}^{2}}{\mathrm{n}_{2}}}<\left|\mathrm{M}_{1}-\mathrm{M}_{2}\right|
$$

ist, was mit 50, $52<65,4$ bei unseren Werten der Fall war.

Wir danken Herrn Oberarzt Dr. PaEtzoț von der UniversitätsHautklinik Gießen und Herrn Werkssanitäter H. NACHTIGALL von den Didier-Werken, Werk-Mainzlar, für das Zurverfügungstellen der Hainproben.

\section{Literatur}

1. Herrmann, R. und K. Rötger, diese Z. 4, 217 (1966). - 2. StAMM, D. und K. RöTGER, diese Z. 4, 220 (1966). - 3. Ruf, E., Z. analyt. Chem. 161, 1 (1958). - 4. Herrmann, R. und C. TH. J. Alkemade, Flammenphotometrie, 2. Aufl. Springer-Verlag, Berlin-
Göttingen-Heidelberg (1960). - 5. Kotter, S., Graph. Tafeln zur Beurteilung statist. Zahlen. Th, Steinkopf-Verlag, Dresden (1943). - 6. KAISER, H. und H. SPECKER, Z. analyt. Chem. 149, 46 (1956). 